\title{
La radioprotection à l'échelle de l'Europe 1992*
}

\author{
L.-J. BRINKHORST **
}

(Manuscrit reçu le 10 juillet 1989)

Les actions de la Communauté européenne dans le domaine nucléaire se basent sur le traité Euratom de 1957. L'objectif principal de ce traité vise à créer les conditions nécessaires à la croissance des industries nucléaires. A son entrée en vigueur en 1958, un de ses buts primordiaux était de favoriser un programme ambitieux de recherche et de développement. L'industrie de l'énergie nucléaire en était à ses débuts. Depuis lors, la production d'énergie nucléaire a connu un développement tel, qu'en 1988, 139 centrales nucléaires étaient en service dans la Communauté. De plus, depuis 1958, le parc des installations nucléaires et la gamme des applications du nucléaire se sont développés d'année en année, si bien qu'aujourd'hui près d'un million de travailleurs communautaires sont régulièrement contrôlés en raison de leur exposition aux rayonnements ionisants, auxquels ils sont soumis dans le cadre de leur activité professionnelle. Celle-ci, dans $80 \%$ des cas, concerne des domaines autres que l'industrie nucléaire.

L'industrie nucléaire de la Communauté a contribué, dans ces dernières années, à réduire notre dépendance vis-à-vis des importations de pétrole et, de façon plus générale, à diminuer la pression sur les ressources pétrolières mondiales. L'expansion du nucléaire a également aidé, dans une certaine mesure, à ralentir la dégradation de l'atmosphère de notre planète et de notre environnement, provoquée par les résidus de combustion des combustibles fossiles.

* Discours d'ouverture du colloque "Radioprotection et maintenance des centrales nucléaires à l'horizon 1992 ", organisé par l'Association belge de radioprotection et la Société française de radioprotection. Bruxelles, 24-26 mai 1989.

** Directeur général, Environnement, Sécurité nucléaire et Protection civile, Commission des Communautés européennes, rue de la Loi 200, Bruxelles, Belgique.

RADIOPROTECTION, VOL. 25 - 0033-8451/1990/3/\$5.00/@Gédim.

Article published by EDP Sciences and available at http://www.radioprotection.org or http://dx.doi.org/10.1051/radiopro/1990026 
Les applications des techniques nucléaires autres que la production d'électricité ont eu aussi un développement remarquable. Dans les hôpitaux des pays communautaires, un patient sur quatre profite des techniques nucléaires soit pour le diagnostic, soit pour la thérapie. Ces techniques nucléaires contribuent, également, de façon importante, à l'évolution de l'agriculture et sont devenues des pratiques courantes dans l'industrie classique.

L'accident survenu à la centrale nucléaire de Tchernobyl, en avril 1986, a de nouveau posé la question de la sécurité nucléaire. II a montré que l'exposition potentielle à la radioactivité concerne toute la population de la Communauté, car elle vit effectivement dans le voisinage plus ou moins large d'une centrale nucléaire. Tchernobyl a été, de loin, l'accident le plus grave de l'industrie nucléaire, et le seul qui ait produit des conséquences sérieuses sur l'environnement. II a montré qu'il n'est plus possible de considérer les centrales nucléaires dans un contexte exclusivement national.

Parallèlement au développement de l'industrie nucléaire, la protection de la santé et de l'environnement revêt une importance essentielle aux yeux des citoyens européens. II existe en eux une inquiétude accrue des risques d'exposition aux rayonnements. Celle-ci a affecté à son tour le développement de l'industrie nucléaire. Des sondages d'opinion montrent qu'il existe, dans plusieurs États membres, une opposition importante de l'opinion publique au développement ou à l'existence même de l'énèrgie nucléaire. Cette opposition est souvent associée à une information déficiente sur l'utilisation des techniques nucléaires, sur les risques dérivés de cette utilisation, et sur les mesures qui existent pour réduire ces risques. Le contraste à cet égard avec d'autres domaines industriels, par exemple celui de l'industrie chimique, est significatif. C'est pourquoi la sécurité nucléaire, la protection radiologique, ainsi que l'information du public, ont, sur ce sujet, un rôle de la plus grande importance.

Mais quel rôle doit jouer la Communauté européenne, en particulier la Commission, dans le domaine de la radioprotection?

Parmi les tâches principales, assignées à la Communauté par le traité Euratom, figurent l'élaboration de normes de sécurité uniformes pour la radioprotection de la population et des travailleurs et la surveillance de leur application.

Le cadre réglementaire pour accomplir cette tâche prévoit un certain nombre de missions incombant aux institutions communautaires et aux Etats membres.

Depuis ses débuts, la Communauté s'est efforcée de fonder son approche de la radioprotection sur des principes scientifiques irréfutables. Cette position a toujours été considérée comme la plus claire et la seule qui puisse garantir le maintien de la conformité entre les directives-normes de base Euratom et les dispositions de protection radiologique prises par les pays non membres de la Communauté.

Le chapitre III du traité Euratom est spécifiquement consacré à la radioprotection, et c'est en vertu de ces dispositions que, depuis 1959, le Conseil 
des ministres de la Communauté a arrêtė des directives successives fixant les normes de base relatives à la protection sanitaire de la population et des travailleurs contre les risques résultant des rayonnements ionisants. Les normes de base actuellement en vigueur datent de 1980 et 1984 .

Dans le souci d'une protection optimale de ses citoyens, la Communauté a procédé, chaque fois que l'évolution des connaissances scientifiques le réclamait, à une adaptation des directives-normes de base. Avant même d'envisager une nouvelle révision, la Commission insiste sur l'importance de maintenir le consensus international dans ce domaine, et sur la nécessité d'une concertation des organisations internationales concernées. Ce consensus est particulièrement important dans le contexte de la réalisation du Marché unique prévu pour 1992, afin d'éviter que des règles de radioprotection différentes ne créent des entraves aux échanges.

Dans beaucoup de secteurs où la Commission a déjà des responsabilités réglementaires, l'arrivée du Marché unique en 1992, avec les perspectives de concurrence, de croissance et de spécialisation qui en résulteront, va introduire de nouveaux défis et problèmes. J'estime que si le consensus que je viens d'évoquer est préservé, la situation dans le domaine de la radioprotection va pouvoir se développer sans trop de difficultés.

Avec l'entrée en vigueur du Marché unique, il est certain que les actions de coopération et les échanges de personnel entre pays vont augmenter. C'est pourquoi, il faut se préoccuper dès maintenant du problème posé par le suivi dosimétrique des travailleurs exposés de catégorie $A$, en particulier celui des travailleurs d'entreprise de sous-traitance ou d'intérim.

Afin d'assurer une protection efficace de cette catégorie de travailleurs, j'ai donc demandé à mes services de préparer un projet de directive sur la protection opérationnelle des travailleurs extérieurs, intervenant dans des installations utilisant des rayonnements ionisants. Pour obtenir une protection efficace de ces travailleurs, le projet de directive prévoit l'utilisation d'une attestation individualisée d'intervention.

Nous pourrions aller plus loin et envisager une éventuelle liaison, au niveau communautaire, des différents systèmes régionaux et nationaux d'enregistrement de doses. Mais avant d'y parvenir, il faudrait mettre en ceuvre une harmonisation préalable des systèmes de suivi dosimétrique et obtenir la reconnaissance et l'approbation mutuelle des services de dosimétrie. Le projet de directive que j'ai évoqué représentera aussi un premier pas dans l'objectif d'une harmonisation européenne.

Les États membres de la Communauté ont le devoir de prendre des dispositions législatives, réglementaires et administratives pour adapter leur propre législation aux normes de base. La responsabilité de la mise en œuvre de cette législation incombe bien, dans chaque État membre, aux spécialistes en radioprotection. Ceux-ci doivent se consacrer à éviter des pratiques dangereuses et prévenir, si besoin, des risques non nécessaires. Mais, en même temps, les spécialistes en radioprotection devraient s'engager plus activement dans des efforts d'information du public afin 
d'expliquer en termes compréhensibles les avantages des applications judicieuses des rayonnements ionisants, ainsi que les mesures de protection associées qui garantissent la sécurité de ces applications.

Après l'accident de Tchernobyl, la Commission a établi en 1986 un programme de travail en vue d'adapter, voire de développer, ses actions en vertu des dispositions du chapitre III du traité Euratom qui concerne la radioprotection des travailleurs et de la population. C'est ainsi qu'elle a, entre autres, muitiplié ses propres efforts d'information parmi lesquels je cite, notamment, la proposition sur les mesures de protection sanitaires applicables et sur le comportement à adopter en cas d'urgence radiologique. Je suis convaincu que l'adoption de cette directive par le Conseil contribuera de façon significative à l'amélioration de l'information du public. Dans ce contexte, je mentionne également la création d'une conférence permanente sur la santé et la sécurité à l'ère nucléaire. Par ailleurs, la Commission a entamé l'élaboration de brochures destinées au public. Elie poursuivra aussi ses initiatives en vue de l'information et de la formation des travailleurs dans le domaine du nucléaire.

L'amélioration de l'information du public est devenue une condition indispensable pour obtenir une certaine objectivité dans le débat sur le nucléaire. Dans le cadre d'un débat objectif, les avantages relatifs présentés par un nucléaire bien géré, comme la compétitivité, l'indépendance économique ou l'impact environnemental, pourraient être dûment appréciés par le public.

Même si l'attention de celui-ci continue à être focalisée sur la sûreté des centrales nucléaires, on a constaté par le passé - l'accident de Goiània en 1987 en est un exemple - qu'un accident dans des applications médicales et industrielles du nucléaire peut avoir aussi de graves conséquences sur le public et influencer sa confiance dans tout ce qui touche le nucléaire. L'application rigoureuse des mesures de radioprotection dans ces applications revêt la même importance que dans la production d'électricité.

Dans la cadre de ce programme de travail, nous avons développé une réglementation communautaire fixant les niveaux admissibles de radioactivité dans les denrées alimentaires ; d'autre part, un système d'échange rapide d'information en cas d'urgence radiologique a été mis en place.

La Commission a développé également des mesures importantes liées au suivi et à l'évaluation de l'impact de la radioactivité dans l'environnement. D'ailleurs, l'article 35 du traité Euratom donne à la Commission le droit d'inspection des installations nationales de surveillance de la radioactivité dans l'environnement, et la Commission étudie actuellement les conditions dans lesquelles ce droit pourrait être appliqué.

En matière de recherche et développement, la Commission gère des programmes importants, à la fois directement dans ses installations du Centre commun de recherche (CCR) et à frais partagès en collaboration avec les institutions scientifiques des États membres. Ces programmes concernent notamment la radioprotection et la sûreté des réacteurs. 
Un problème soulevé par l'affaire "Transnuklear", lequel a, notamment, amené le Parlement européen à instituer, en janvier 1988, une commission d'enquête sur la manutention et le transport de matières nucléaires, est celui du renforcement du suivi administratif des transferts de déchets radioactifs, à l'instar de ce qui est imposé par une directive du Conseil relative aux déchets dangereux. Le programme de travail de la Commission prévoit qu'une proposition à cet effet, basée sur l'article 31 du traité Euratom soit soumise dans les prochains mois aux institutions communautaires concernées.

Toutes ces actions sont de la plus grande importance parce que la Commission est d'avis que le nucléaire ne pourra continuer à jouer un rôle important en Europe que si les citoyens ont la conviction d'avoir leur sécurité et leur santé assurées. Dans ce contexte, une réglementation claire en radioprotection, s'appuyant sur des bases scientifiques confirmées, et son application rigoureuse constituent des conditions nécessaires pour la meilleure protection possible.

Je considère qu'il est du plus grand intérêt pour la Communauté, et pour chaque État membre, de faire un effort dans l'application des dispositions du chapitre III du traité Euratom afin d'améliorer la protection de la santé des citoyens européens.

La convocation de ce colloque se situe à un moment opportun, et j'espère que les objectifs qui ont été fixés par les organisateurs seront atteints. Le colloque doit servir, comme je l'entend, de lieu de rencontre pour un échange d'opinions, au niveau européen, sur les fruits de l'expérience acquise et puisse-t-il être pour vous, en tant que spécialistes en radioprotection, une occasion de réfléchir sur les solutions possibles aux problèmes posés et sur l'influence du Marché unique de 1992 à cet égard.

La radioprotection à l'échelle de l'Europe de 1992 est un véritable défi pour tous ceux qui ont des responsabilités réelles dans ce domaine. En vous exprimant mes meilleurs vœux pour une pleine réussite de votre réunion, je voudrais assurer les membres de vos associations que la Commission continuera à assumer les responsabilités qui lui ont été attribuées par le traité Euratom, en particulier dans le domaine de la radioprotection. Mais, comme par le passé, elle compte sur une collaboration constructive avec les services nationaux chargés de la radioprotection, et aussi sur celle des associations nationales de radioprotection que vous représentez ici.

\section{RÉFÉRENCES}

Traité instituant la Communauté européenne de l'énergie atomique (EURATOM), Rome, 25 mars 1957, Bruxelles: Communautés européennes, 1957. 